\title{
Anatomical studies of the collateral blood supply to the brain and the retina
}

\author{
J. SZAPIRO AND H. PAKULA
}

From the Neurosurgical Clinic and the Department of Anatomy, Medical Academy, Lódź, Poland

A turning point in the attitude to cerebrovascular disorders has become apparent in the last decade and is related in some degree to the growing interest in the arterial territory situated between the aortic arch and the circle of Willis. This report deals with anatomical studies concerning one of its main fragments which has been called by one of the present authors (Szapiro, 1960) the anterior aorto-cerebral circle or the carotid circle (Fig. 1a, left upper quadrant). This area is a common site for various pathological processes such as thromboses, arteriosclerotic lesions narrowing the lumen of the arteries, aneurysms, developmental anomalies, and carotid sinus hypersensitivity. Also, in this region many procedures are performed, such as angiography, carotid ligation, thrombembolectomy, bypass graft, and denervation of the carotid sinus. Some of these pathological processes and procedures may contribute to cerebrovascular insufficiency and certain operations tend to relieve these disorders. Its usefulness is also due to the fact that the carotid circle is formed for the most part by vessels which are nearly symmetrical and have one 'dead point' situated in physiological conditions in the anterior communicating artery. The accessibility of the carotid arteries in the neck to palpation and compression makes it possible to study the behaviour of the collateral circulation by causing its temporary breakdown or mobilization by means of the carotid compression test. This control may be studied not only through observation of the patient's behaviour but also by such ancillary methods as angiography, electroencephalography, and ophthalmodynamometry.

The external carotid arteries with their branches and their anastomoses must be studied together with the internal carotid system in considering the carotid circle, and the strategic position of the ophthalmic arteries is obvious (Fig. 1a). Clinical examination of the terminal branches of the ophthalmic arteries by means of ophthalmodynamometry is known to be useful in the diagnosis of carotid occlusion and may also provide information on collateral blood flow passing through the connexions between the internal carotid arteries, that is, through the anterior part of the circle of Willis.

Anatomical post-mortem studies are convenient कै for investigating the anastomoses between internal $\overrightarrow{0}$ and external carotid systems with special reference $\overrightarrow{-}$ to the ophthalmic artery. Direct blood supply to the $\omega$ brain from the external carotid system by the ophthalmic arteries is fully active in phylogenesis and ontogenesis. It loses its significance during extrauterine is human life and regains it only in certain cases of or ipsilateral internal carotid artery thrombosis, prob ably where the more natural channels of collatera $\vec{A}$ circulation are ineffective.

Anatomical studies related to the problem of the carotid circle were initiated by Elschnig (1893. Many years later they were renewed by Walsh and King (1942) and also by Shea (1956), who even attempted to analyse this problem statistically. 再 these experiments the role of the external caroti $\%$ artery as a potential source of the blood supply was $\omega$ elucidated and can often be confirmed by angiography.

Further studies of certain details concerning this subject seemed to be justified, and in our anatomical investigations, referred to in detail in a separate $\mathbb{D}$ report (Szapiro, Goetzen, Świetliczko, Pakuła, and Głuszcz, 1962) we have used, while injecting the arteries, a method of eliminating the possible channels of the collateral circulation. Usually the internal carotid artery was excluded, and a latex medium prepared according to Goetzen's method (1957) or, alternatively preserved blood was injected into the ipsilateral external carotid artery or into its branches. Each experiment was performed in at least two variants. In the first the connexion between the internal carotid arteries was patent and in the second it was blocked by a clip. The main purpose of these experiments was to fill both ophthalmic $\frac{D}{2}$ arteries with the injected substance through various extracranial routes and to determine the difference $N$ in time between the filling of each. To complete the study several experiments were carried out in order to investigate the possibility of filling the internal 


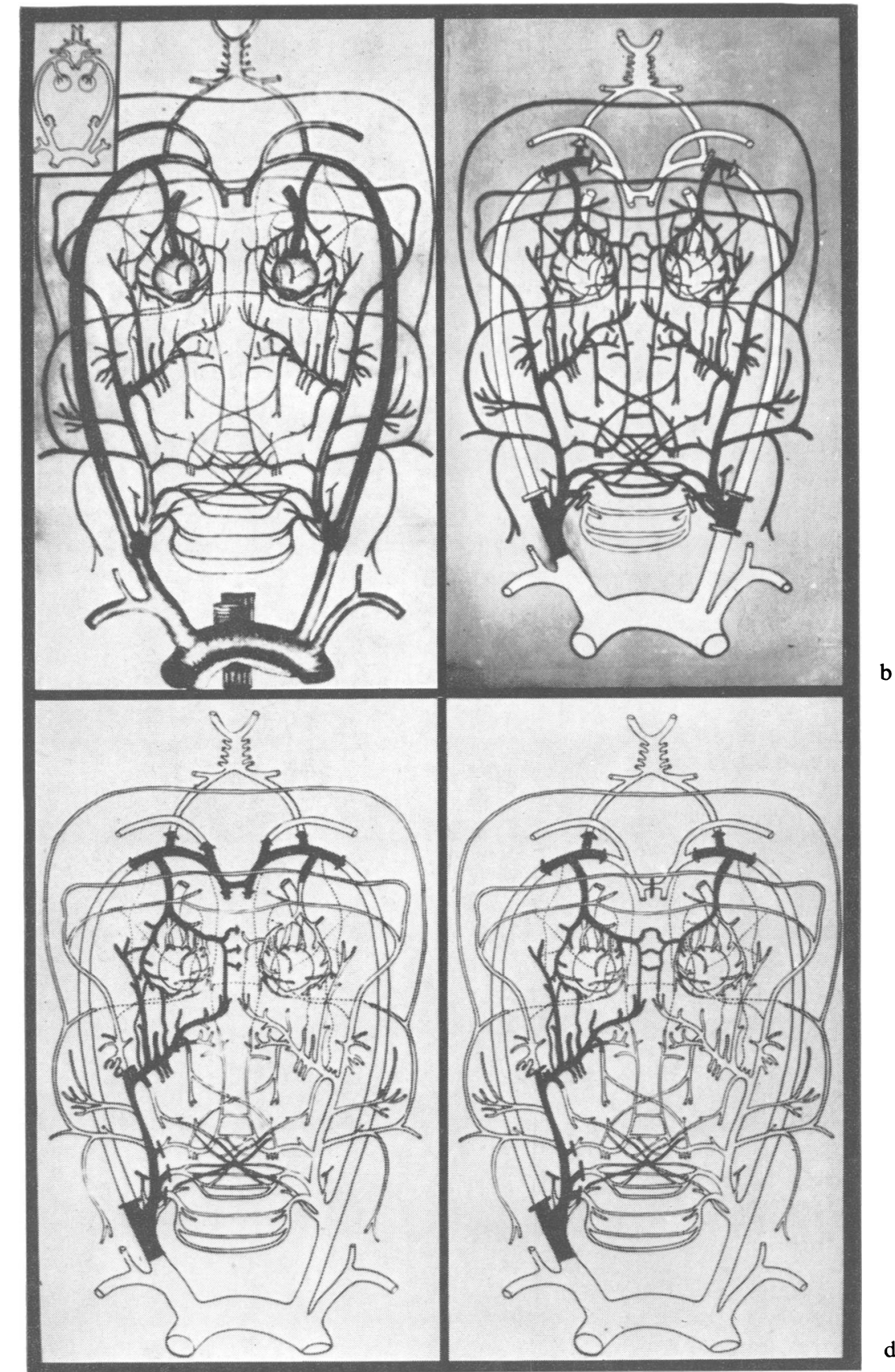

FIG. 1a. The anterior aorto-cerebral circle to show the anastomoses between both external carotid arteries and between each external carotid artery and the homolateral ophthalmic artery.

FIG. 1b. The latex medium was injected into the right external carotid artery. The right internal carotid artery was previously ligated in the neck. Furthermore a clip was set on the left internal carotid artery intracranially between the ophthalmic artery and the posterior communicating artery. The medium obviously reached both ophthalmic arteries by an extracranial route.

FIG. 1c. The latex medium was injected into the external carotid artery. Some of its branches, and among them the superficial temporal artery and the facial artery, were previously clipped at their beginning, allowing most of the medium to flow into the maxillary artery. The medium reached both ophthalmic arteries. d

FIG. 1d. The same as in Fig. 1c. An accessory clip was set on the anterior communicating artery to exclude the possibility of the opposite ophthalmic artery being filled by way of the anterior part of the circle of Willis. The medium reached both ophthalmic arteries. 
carotid artery from the ipsilateral external carotid artery by channels other than the ophthalmic artery. A few corrosive experiments were added. Radiographic verification was only performed occasionally. Unfortunately, a mechanical injector was not available. The number of experiments, including also the studies of the posterior aorto-cerebral circle, did not exceed 50, so no statistical conclusions could be drawn: in each particular group one to four experiments were performed.

The first group of experiments was, in fact, a repetition of studies reported by former authors, and it was confirmed that the medium injected into one external carotid artery may reach the cerebral arteries of both cerebral hemispheres and fill both ophthalmic arteries as well. We could not fully confirm this last detail, so in the next experiment (Fig. 1b), before the medium was injected into the right external carotid artery, a clip was placed on the left internal carotid artery intracranially between the ophthalmic artery and the posterior communicating artery. In this way any possible filling of the left ophthalmic artery through the arteries of the circle of Willis was excluded. It was found that the medium still reached both ophthalmic arteries obviously through the branches of the external carotid arteries.

In the next group of experiments the latex medium was injected into one of the branches of the external carotid artery, namely, into the superficial temporal, then to the maxillary and to the facial arteries. The medium reached both ophthalmic arteries in all these experiments. Figure 1c represents this fact schematically. This result was not prevented by placing a clip on the anterior communicating artery and the medium reached the cerebral vessels of both hemispheres apparently through anastomoses of both external carotid arteries in the neck and face (Fig. 1d).

Concerning the structural features of these anastomoses, corrosive experiments have shown (Figs. 2a and 2b) that some of these connexions are characterized by an extensive continuum of vessels formed by plexuses of small arteries of the pterygopalatine and temporal fossa, of the inferior orbital fissure, nasal mucosa, nasal septum, ethmoidal cells, and the maxillary sinus. On the basis of the morphological features of these plexuses, i.e., their density, the cross section of the vessels and their polystriatal structure, it seems that they must be considered as possible channels for the collateral blood stream flowing from the external carotid artery to the ipsilateral as well as to the opposite ophthalmic artery. Among the ipsilateral anastomoses a particularly important role seems to be played by the maxillary artery (Figs. $2 a$ and $2 c$ ) but the significance of the superficial temporal and the facial arteries in this respect should not be under-

\begin{tabular}{lcc} 
& TABLE I & \\
Injected Artery & Filling of Ophthalmic Arteries (sec.) \\
\cline { 2 - 3 } & Ipsilateral & Heterolateral \\
\hline Intracranial route & & \\
Internal carotid & 2 & 6 \\
Extracranial route & & \\
External carotid (superior thyroid & & \\
excluded) & +48 & +78 \\
Maxillary & +7 & +30 \\
Facial & +12 & +100 \\
Superficial temporal & +14 & + \\
Occipital & +15 & - \\
Posterior auricular & - & - \\
Lingual & +90 & +56 \\
Ascending pharyngeal & +95 & +75 \\
Superior thyroid & +72 & +60
\end{tabular}

estimated. Of the heterolateral anastomoses, attention should be paid to the maxillary arteries (Figs. $2 \mathrm{~b}$ and 2c). Table I sets out results of experiments which show both the possibilities of filling of the ipsilateral and opposite ophthalmic artery by the medium injected into various branches of the external carotid artery and the respective time differences.

All our findings should be confirmed in a larger series but even so they can serve as a general guide The same applies to the recently suggested possibilit of filling of the internal carotid artery from the 응 external carotid artery by routes other than th@ ophthalmic, which finding was indeed observed ir one of our experiments.

\section{CONCLUSIONS}

Our studies have shown that there are numerous possible channels of collateral circulation from the external carotid artery to the brain and retina. They reflect not only the physical laws of fluid flow in patent tubes but they also provide a useful indication of the blood flow in certain pathological conditions. They have been partly verified by angiographic data and by correlation with the clinical experience of neurologists and neurosurgeons. However, morphological facts of the collateral circulation are not equivalent with its real adequacy. Therefore, our general conclusion depends only on the difference between the nature of Heubner's investigations and our own. Heubner in 1874 succeeded in filling all the cerebral arteries by means of injecting the medium into one cerebral artery unconnected with the circle of Willis. In our studies the same results could be achieved by injecting the medium into one extracranial artery, which proved also to be an indirect source of the brain supply. The shift of interest in the problem from intracranial to extracranial components seems to be symptomatic of the ever-changing views on this difficult subject. 


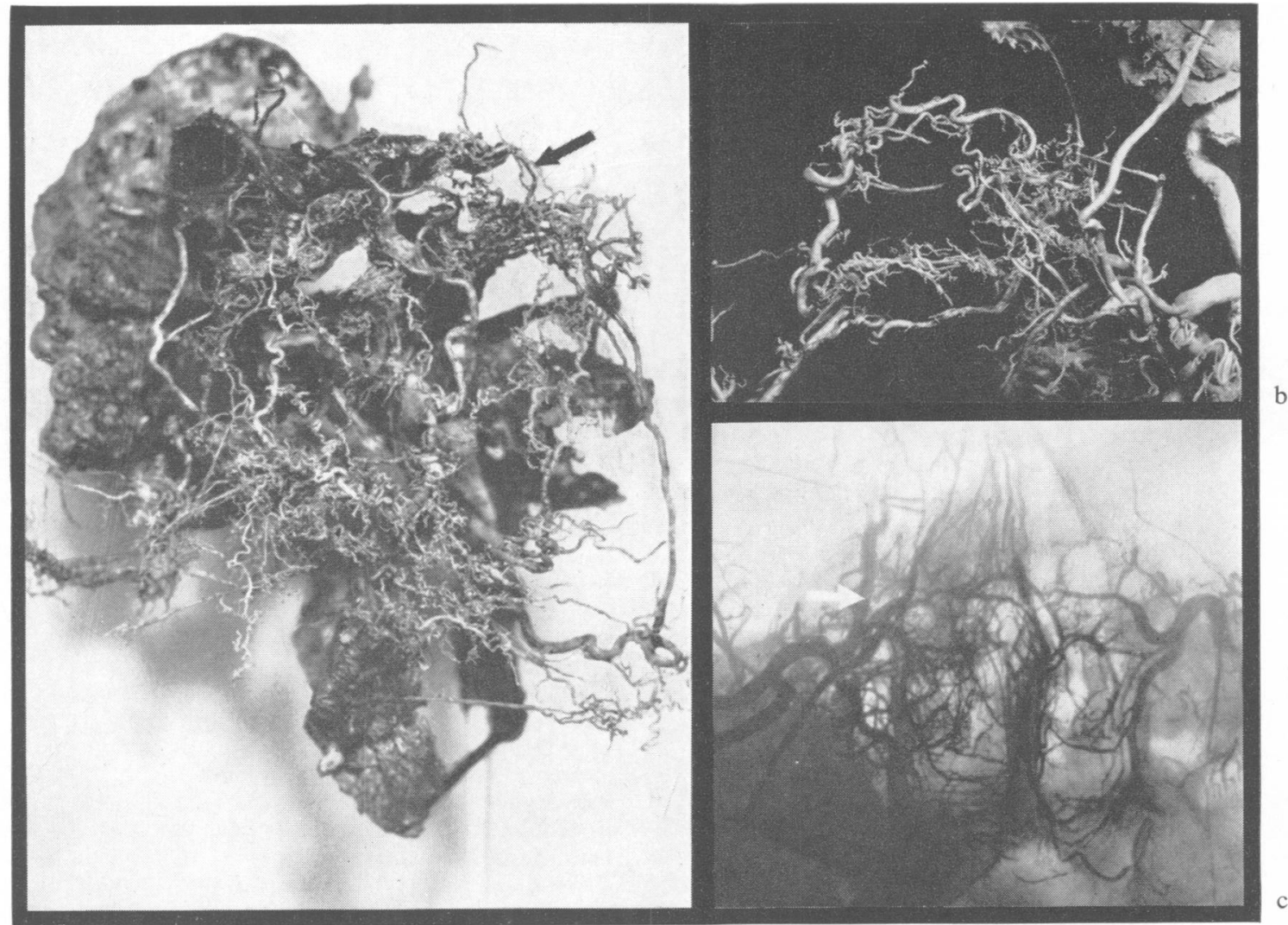

FIG. 2a. The photograph shows a fragment of the anastomoses between branches of the external carotid artery and the homolateral ophthalmic artery, as shown by the corrosive experiment. White arrows are anastomoses between branches of the maxillary artery and the ophthalmic artery and the black arrow anastomoses between branches of the facial artery and of the ophthalmic artery.

FIG. 2b. The photograph shows a fragment of the anastomoses between both external carotid arteries, as shown by the corrosive experiment.

FIG. 2c. The radiological picture of some of the anastomoses between both maxillary arteries. The ophthalmic artery (white arrow) was filled by medium injected into homolateral maxillary artery.

\section{REFERENCES}

Elschnig, A. (1893). Albrecht v. Graefes Arch. Ophthal., 39, pt. 4, p. 151.

Goetzen, B. (1957). Pol. Tyg. lek., 12, 296.

Heubner, O. (1874). De luetische Erkrankung der Hirnarterien. Vogel, Leipzig.
Shea, M. (1956). Brit. J. Ophthal., 40, 497.

Szapiro, J. (1960). In the Monography: $Z$ zagadnien fizjopatologii krażenia krwi w mózgu. Lódż. P.Z.W.L.

_- J. Goetzen, B. Swietliczko, I., Pakuła, H., and Głuszcz, A. (1962). Arch. Acad. Med. Lódż.

Walsh, F. B., and King, A. B. (1942). Arch. Ophthal. (Chic.), $27,1$. 